\title{
Cubic ice Ic without stacking defects obtained from ice XVII
}

\author{
Leonardo del Rosso, Milva Celli, Francesco Grazzi, \\ Michele Catti, Thomas C. Hansen,
} A. Dominic Fortes and Lorenzo Ulivi

\section{Published version information}

Citation: L del Rosso et al. "Cubic ice Ic without stacking defects obtained from ice XVII." Nature Materials, vol. 19 (2020): 663-668.

DOI: $\underline{10.1038 / s 41563-020-0606-y}$

This is a post-peer-review, pre-copyedit version of an article published in Nature Materials. The final authenticated version is available online at the DOI above.

This version is made available in accordance with publisher policies. Please cite only the published version using the reference above. This is the citation assigned by the publisher at the time of issuing the AAM. Please check the publisher's website for any updates. 


\title{
Cubic ice Ic without stacking defects obtained
}

\section{from ice XVII}

\author{
Leonardo del Rosso, ${ }^{* \dagger}{ }^{\dagger}$ Milva Celli, ${ }^{\dagger}$ Francesco Grazzi, ${ }^{\dagger}$ Michele Catti, ${ }^{\ddagger}$ Thomas \\ C. Hansen, "Andrew Dominic Fortes, ${ }^{\S}$ and Lorenzo Ulivi*, ${ }^{*}$ \\ $\dagger$ Consiglio Nazionale delle Ricerche, Istituto di Fisica Applicata "Nello Carrara", \\ via Madonna del piano 10, I-50019 Sesto Fiorentino, Italy \\ $\ddagger$ Dipartimento di Scienza dei Materiali, Università di Milano Bicocca \\ via Roberto Cozzi 55, I-20125 Milano, Italy \\ 9Institut Laue-Langevin (ILL), 71 avenue des Martyrs, 38000 Grenoble, France \\ $\S I S I S$ Neutron and Muon Facility, Rutherford Appleton Laboratory, Harwell Science and \\ Innovation Campus, Chilton, Oxfordshire OX11 OQX, U.K. \\ E-mail: I.delrosso@ifac.cnr.it; I.ulivi@ifac.cnr.it
}

December 20, 2019 
Among the over eighteen different forms of water ice, only the common hexagonal phase and the cubic phase are present in nature on Earth. Nonetheless, it is now widely recognised that all samples of "cubic ice" discovered so far do not have a fully cubic crystal structure and instead are stacking-disordered forms of ice I (namely ice Isd), which contain both hexagonal and cubic stacking sequences of hydrogen-bonded water molecules. Here we describe a method to obtain large quantities of cubic ice Ic with high structural purity. Cubic ice Ic is formed by heating a powder of $\mathrm{D}_{2} \mathrm{O}$ ice XVII obtained from the annealing of pristine $\mathrm{C}_{0}$-hydrate samples under dynamic vacuum. Neutron diffraction experiments performed on two different instruments and Raman spectroscopy measurements confirm the structural purity of the cubic ice Ic. These findings contribute for a better understanding of ice I polymorphism and the existence of the two natural ice forms.

Ice is omnipresent in nature, on the surface of the Earth, in the atmosphere, and on celestial bodies. ${ }^{1,2}$ The phase diagram of solid water presents a large complexity, with a plethora of structures which are stable, or metastable, at different pressure - temperature conditions. ${ }^{3-5}$ At ambient pressure, liquid water freezes forming ice Ih, which is the stable phase in a vast region of temperature and pressure and has an hexagonal crystal structure. A different phase was obtained at ambient pressure already long time ago by water vapour deposition at low temperature, ${ }^{6}$ and was considered to be cubic on the basis of its electron diffraction pattern. Later, the same phase was formed by the transformation of amorphous ice, obtained by depositing vapour at about $110 \mathrm{~K}$, with increasing temperature above $140 \mathrm{~K},{ }^{7}$ and, in larger quantities, by the back-transformation, at ambient pressure and low temperature, of crystalline high-pressure ice polymorphs. ${ }^{8-11}$ More recently ice with signs of cubic structure were produced by other methods, as the freezing of nano-droplet, ${ }^{12}$ the dissociation of gas hydrates, ${ }^{13,14}$ and the freezing of water in confined geometry. ${ }^{15}$ It is now clear, however, that these solids are not structurally cubic ice, since they present always 
different amounts of stacking defects, which depend on the starting material. ${ }^{16-18}$ This phase, indicated as Isd, is therefore named stacking-disordered ice. ${ }^{17}$ Experimental results, as the observation of different kinds of halo in polar regions, suggest that ice Isd is present in the atmosphere, as stacking disorder influences the crystal shape and orientation. ${ }^{19-21}$ Amorphous and stacking-disordered ice plays also important roles for the stability of comets. ${ }^{22}$

A representation of the stacking defects of ice Isd, compared with ice Ih and ice Ic, is reported in Fig. 1. The $\mathrm{O}$ atoms of water molecules in all ice I phases are arranged in pairs, bound parallel to the crystallographic $c$ axis, which is also the stacking direction. These pairs lay on hexagonal close packed layers, which are piled up to form the three dimensional crystal. If one does not consider the hydrogen atoms, a close analogy can be drawn with monoatomic metals, where stacking sequences of the type ABABAB... or ABCABC... give rise to hexagonal (Ih) or cubic (Ic) three-dimensional symmetry, respectively. Stacking defects of the Isd structure correspond, for example, to insertion of slices with hexagonal sequence into the cubic one. Given a sequence, the character of each layer, cubic or hexagonal, can be determined unequivocally by looking at the preceding and following layers. The cubicity of a sample, i.e. the proportion of cubic stacking sequences, ${ }^{16}$ can be determined by the analysis of accurate diffraction measurements. Ice Isd has been studied in the past in great detail, ${ }^{23}$ even if a cubicity higher than $80 \%$ has never been observed to date.

We have prepared structurally pure ice Ic by the transformation of a powder of ice XVII at ambient pressure by increasing temperature. Ice XVII is a metastable phase of ice, obtained from the high-pressure hydrogen filled ice in the $\mathrm{C}_{0}$-phase. ${ }^{24,25}$ This low density solid water phase is highly porous, and, unique among the various stable and metastable phases of ice, exhibits a structure comprising only pentagonal rings of water molecules. ${ }^{25,26}$ Ice XVII can be maintained at ambient pressure only up to about $130 \mathrm{~K}$, above which it undergoes a phase transition similar to that mentioned above for the amorphous ${ }^{7}$ and high-pressure crystalline $^{8,9,11}$ forms. Whilst the end-product of all of these transitions, above $200 \mathrm{~K}$, is the ordinary hexagonal form of ice (ice Ih), the remarkable difference between ice XVII and the 
other forms is the nature of the intermediate state, where, instead of stacking-disordered ice, we find a structurally-pure form of cubic ice.

This surprising outcome arises from two different neutron diffraction experiments performed, respectively, on HRPD at ISIS, (UK) and on D20 at ILL (F), during which we have studied the transformation of metastable ice XVII towards a stable phase. The experimental procedure was very similar in the two cases. In both experiments the pure $\mathrm{D}_{2} \mathrm{O}$ ice XVII sample, obtained after the completion of the annealing treatment on the $\mathrm{C}_{0}$-hydrate pristine sample, was heated under dynamic vacuum, measuring the diffraction patterns at constant temperature intervals (typically, $5 \mathrm{~K}$, in the HRPD experiment or $10 \mathrm{~K}$, in the D20 experiment), in the range 110-180 K. For further details see the Method section.

The main results of both experiments are summarized in Fig. 2, where the most representative raw HRPD (a) and D20 (b) diffraction patterns are reported. In both cases a clean ice XVII diffraction pattern ( $P 6_{1} 22$ symmetry group) is measured at low temperatures, below about $150 \mathrm{~K}$. On heating, a phase transition is observed between 150 and $160 \mathrm{~K}$ by the appearance of new diffraction peaks belonging to a different crystallographic phase; these were successfully indexed with the cubic symmetry $F d \overline{3} m$. Other details of this phase transition are reported in the Supplementary Information (SI, Supplementary Note 1). The refined parameters, obtained with the Rietveld method ${ }^{27}$ using either the GSAS, ${ }^{28}$ or the the FullProf software packages, ${ }^{29}$ are listed in Table 1.

The diffraction patterns recorded above $160 \mathrm{~K}$, that present only the cubic ice reflections, indicate the completion of the transition from ice XVII to ice Ic. These are reported in Fig. 2c and $2 \mathbf{d}$ for the experiments on HRPD and D20, respectively. The high degree of structural purity of ice Ic, with no detectable sign of stacking disorder, is demonstrated by the absence of any hexagonal ice Ih reflections and diffuse scattering between the Bragg peaks in the whole diffraction patterns and by the good quality of the fit, which is characterized by a weighted profile agreement index $R_{w}$ equal to $4.1 \%$ for the D20 data and $4.4 \%$ for the data taken on HRPD. Indeed, also very weak reflections as (311) and (400) are observed, (see 
Suppl. Fig. 2) with an intensity matching the expected one for a pure cubic crystal. Even the presence of any fraction of possible amorphous ice phases in the sample can be totally excluded from the observation that the continuous background intensity remains very small after the transition from ice XVII to ice Ic at about $150 \mathrm{~K}$.

Producing ice Ic with such a structural purity surely represents a major breakthrough. What is generally obtained is instead ice Isd, whose degree of stacking disorder can vary substantially as a function of the preparation method and history of the sample. ${ }^{16}$ The diffraction pattern in this case may present symmetry-forbidden reflections, and can be modelled and fitted with the one pertaining to stacking-disordered ice with variable cubicity. ${ }^{18,23,30-32}$ Numerous examples of diffraction patterns commonly obtained for ice Isd, synthesised by different routes, are reported in Fig. 3 of Ref. 18. So far, the highest cubicity claimed experimentally is $\approx 80 \%$, even though derived by an analysis of only a portion of the diffraction pattern. ${ }^{33}$ In the SI (Supplementary Note 2) we compare the portion around $Q=1.7 \AA^{-1}$ of the diffraction pattern obtained in our experiments with that of some instances of ice Isd among those having the highest degree of cubicity obtained to date.

From the Rietveld refinement of the HRPD data we have derived the behaviour of the unit cell parameters as a function of the temperature, during and after the transition from ice XVII to ice Ic. The density of ice XVII and ice Ic $\left(\mathrm{D}_{2} \mathrm{O}\right)$ is reported in Fig. 3. The data collected before and after the transition were refined with a single phase (i.e. space group $P 6_{1} 22$ and $F d \overline{3} m$ for ice XVII and Ic, respectively), while the data collected during the transition $(150$ and $155 \mathrm{~K})$ were refined with both phases. The lattice parameters obtained at these two transition temperatures might be affected by some systematic uncertainty due to the non-homogeneous filling of the sample can by the two phases. $\mathrm{D}_{2} \mathrm{O}$ ice Ic displays a slightly lower density than that measured for ice Ih. The ice Ih - ice Ic positive density difference is larger than the statistical uncertainty derived from the Rietveld fit, but we cannot exclude a systematic uncertainty, which presumably may be of the same order of magnitude of the difference between the two determinations for ice Ic at $160 \mathrm{~K}$. 
We have also investigated the structural transition of ice Ic into the stable ice Ih by performing a dedicated neutron diffraction experiment on D20 at ILL. In this case the sample cell was not connected to a vacuum pump but sealed (see Methods for details). Diffraction data from a sample of ice Ic, prepared offline from ice XVII, were collected while performing a slow $(0.12 \mathrm{~K} / \mathrm{min})$ temperature ramp from $150 \mathrm{~K}$ to $230 \mathrm{~K}$. In this case, thanks to the high signal/noise ratio of these data, a tiny feature at $2 \theta=36.36^{\circ}$ is visible on an expanded vertical scale in the diffraction patterns even in the first measurements, below $150 \mathrm{~K}$. This is an indication that, in this case, ice XVII did not converted totally into pure ice Ic, probably because the starting ice XVII sample was not perfectly clean from absorbed gas molecules, and these could not be released during the warming cycle because of the sealed vanadium cell. An estimation of the amount of the stacking disorder present in this sample has been done by means of the computer program DIFFaX, ${ }^{36}$ which is freely available. This program calculates diffraction intensities from crystals that contain coherent planar defects, such as stacking faults, as in our case. We have fitted the measured diffraction pattern around the (111) Bragg reflection, which is the only region where some sign of disorder is detected, adjusting in succession the disorder parameters. We obtain a good agreement with a cubicity around $95 \%$. Details of the parameters used in the computation and a figure depicting the results are reported in the SI (Supplementary Note 3).

The diffraction patterns, measured as a function of temperature, have been analysed to follow the transition from ice Ic to ice Ih. We have estimated the growing fraction of ice Ih considering it proportional to the normalized intensities of each of five representative ice Ih Bragg reflections (see Method section). In this procedure, we have assumed zero as the starting ice Ih proportion and $100 \%$ as the final proportion. We favour this method compared to the refinement of each diffraction pattern because of the presence of a small amount of stacking faults in the starting material, which cannot be properly described with a two phases Rietveld fit, with both ice Ic and Ih. The result for the ice Ih fraction is presented in Fig. 4. The sample starts to modify at about $185 \mathrm{~K}$, when the Ih fraction starts to grow, 
reaching $100 \%$ slightly above $215 \mathrm{~K}$. As a matter of fact, attempting a two phases Rietveld refinement of data taken below $200 \mathrm{~K}$, and calculating from them the ice Ih fraction, one obtains values above the reported curve, an indication that the fitting procedure interprets incorrectly the stacking faults as presence of a fraction of ice Ih. Above $200 \mathrm{~K}$ the two phases Rietveld fit converges, giving an estimation of the fraction of ice Ih in agreement with that calculated from the Bragg reflection intensities. At any rate, these data demonstrate that no intermediate phase is formed during the Ic - Ih transition. In the SI we report a contour plot representing all the diffraction data measured during the transformation from ice Ic to ice Ih. (Supplementary Note 4)

We have also performed a Raman spectroscopy study to detect the effects of the two phase transitions on the spectrum. We used $\mathrm{H}_{2} \mathrm{O}$ for this experiment, confident that isotopic differences at these temperatures can lead to a tiny difference in the phase line but not in the crystal structure. The Raman spectra of the two phases, ice XVII and ice Ic, present marked differences, both in the lattice modes $\left(150-350 \mathrm{~cm}^{-1}\right)$ and OH-stretching region (3000-3500 $\mathrm{cm}^{-1}$ ). In the first region (Fig. 5a) one observes in ice Ic a clear shift of the main peak towards higher frequency, and the appearance of a second peak at about $315 \mathrm{~cm}^{-1}$. As a consequence, the shape of the whole band changes markedly. The OH-stretching Raman band (Fig. 5b) shows a clear softening by more than $25 \mathrm{~cm}^{-1}$, while its shape does not change much. This finding is in agreement with the increase of density and, consequently, decrease of the $\mathrm{OH} \cdot \mathrm{O}$ bond length, as already observed in the ice for different compounds ${ }^{37}$ or, for the same structure, as a function of thermodynamic conditions. ${ }^{38,39}$

We have studied the kinetics of the ice XVII - ice Ic transition monitoring the frequency of the $\mathrm{OH}$-stretching band as a function of either temperature or time, in two subsequent experiments. In the first one, the temperature of the sample was slowly increased $(0.1$ $\mathrm{K} / \mathrm{min}$ ) from $120 \mathrm{~K}$ to $178 \mathrm{~K}$, thus allowing to determine the temperature of the structural transformation. The central frequency of the most intense peak of the OH-stretching band, fitted as a Lorentzian (see Method section) is reported as a green line in Fig. 5c. The 
transformation to ice Ic, marked by the large frequency decrease $\left(22.1 \mathrm{~cm}^{-1}\right)$ begins slightly below $139 \mathrm{~K}$, and has it maximum rate at $144 \mathrm{~K}$. The slope of the $\mathrm{OH}$ frequency increase with temperature is slightly larger in ice Ic $\left(0.314 \mathrm{~cm}^{-1} / \mathrm{K}\right)$ than in ice XVII $\left(0.229 \mathrm{~cm}^{-1} / \mathrm{K}\right)$. The transformation appears to be complete in about $110 \mathrm{~min}$, during which the temperature has increased by $11 \mathrm{~K}$. The kinetics of the transformation at one fixed temperature (orange lines in Fig. 5c, 5d), namely $T=139.5 \mathrm{~K}$ (chosen just above the temperature at which a detectable spectral change has been observed in the first experiment) is studied in the second measurement. As expected, in this case the transformation is slower. The time it takes to complete the transformation can be estimated at about $7 \mathrm{~h}$. The transformation process can be also efficiently monitored by observing the width of the first peak versus time (Fig. 5d). The measured broadening during the transition (see also Supplementary Note 5) is obviously due to the coexistence of the two phases during the structural rearrangement, which lasts, in the first case, about $200 \mathrm{~min}$. The kinetic series measured at $T=139.5 \mathrm{~K}$ evidences a much slower change, with a long time tail in the roughly exponential decay of the line width, with a time constant $\tau \simeq 220 \mathrm{~min}$. This is evidence of a very slow structural relaxation process. In any case, the apparent discrepancy of the transition temperature with respect to the diffraction data is due to the faster heating rate applied in the neutron experiment.

Finally, by using Raman technique, we also explored the Ic - Ih transition, measuring, on the same sample, the $\mathrm{OH}$ vibration Raman spectrum as a function of temperature. In analogy with the results of a recent paper, ${ }^{40}$ one might observe, going from Ic to Ih, a sensible down-shift of the peak frequency, as it happens for ice Isd and an overall narrowing of the same peak. We have analysed the spectra recorded between $180 \mathrm{~K}$ and $230 \mathrm{~K}$, determining the peak frequency with a reproducibility of $\pm 0.5 \mathrm{~cm}^{-1}$, and we did not observe any sensible frequency jump during the temperature ramp. Comparing the spectra measured at low temperature on the same sample at the beginning (ice XVII) and after subsequent transformation to ice Ic and ice Ih (Fig. 5a, 5b) one notice clear features that distinguish ice XVII from the other two, while the differences between ice Ic and ice Ih are very subtle. The 
frequency position of the maximum of the $\mathrm{OH}$-stretching band is the same within error $(0.2$ $\mathrm{cm}^{-1}$ ), and only the width of this band is slightly narrower for ice Ih. The lattice band has a somewhat different shape, with a narrower low-frequency feature for ice Ih. From these data it appears that a spectroscopic identification of the type of ice, Ih or Ic, would be very difficult, unless spectra from both phases are recorded and compared.

In conclusion, the result here reported represents a milestone towards the complete characterisation of the ice polymorph I. A recent report describes a similar result obtained by degassing a different high pressure phase of hydrogen hydrate. ${ }^{41}$ While generally ice Isd, with higher or lower cubicity, is produced during the route to stability heating at ambient pressure different metastable phases of ice, it is interesting to underline that starting from a very open structure, as ice XVII, high purity ice Ic is obtained. The low density and the free space between molecules in the starting material, which leaves freedom to rearrangement, is likely to be one of the reasons why a purer phase is achieved.

The new route to obtain pure cubic ice in large quantities opens new perspectives for the clarification of fundamental questions in ice physics. In the future, the specific heat of real ice Ic will be measured, which, together with the Ic - Ih transformation enthalpy, will enable the experimental determination of its free energy, and to resolve the issue of relative stability of the two polytypes of ice present at ambient pressure. This free energy difference is too low to be calculated by the computational methods available at present. ${ }^{42,43}$ Precise structural measurements on good quality ice Ic samples will determine its thermal expansion, and clarify if there is a region of negative expansivity similar to hexagonal ice. In addition, new studies will investigate if hydrogen ordered phases of this same structure exist, in analogy with many other phases of ice, ${ }^{4,44}$ and if cubic ice undergoes at high pressure the same phase transformations as hexagonal ice, or it follows a different route. 


\section{Acknowledgements}

Neutron beam time at ISIS and ILL is gratefully acknowledged, on the basis of the agreement of the CNR (Italy) with STFC (U.K.) and ILL (France) concerning collaboration in scientific research. L. Ulivi and M. Celli acknowledge the PRIN project ZAPPING, No. 2015HK93L7, granted by the Italian MIUR (Ministero dell'Istruzione, dell'Università e della

Ricerca) supporting their research in high-pressure materials science. L. Ulivi, M. Celli and L. del Rosso acknowledge the support from the Fondazione Cassa di Risparmio di Firenze under the contract ICEXVII. ISIS Pressure and Furnaces section and the Electronics section were vital for setting up with gas-handling system and in-situ sample heating for the HRPD experiment. Technical support by Andrea Donati (IFAC-CNR) for the setting up of the high pressure autoclave for the synthesis of the samples is gratefully acknowledged.

\section{Author contribution}

This work is the result of a common effort to which all authors contributed, in particular: L. del Rosso and M. Celli for the synthesis of the samples; M. Celli, L. del Rosso and L. Ulivi for the Raman experiment; M. Catti, L. del Rosso, L. Ulivi and T. C. Hansen for the experiment at ILL; L. del Rosso, F. Grazzi and A. D. Fortes for the experiment at ISIS, RAL; M. Celli and L. Ulivi for the Raman data analysis; M. Catti, L. del Rosso, F. Grazzi and A. D. Fortes for the diffraction data analysis; L. Ulivi, L. del Rosso and M. Celli, for the writing of the manuscript. All the authors read and corrected the manuscript.

\section{Competing interests}

The authors declare no competing interests. 


\section{References}

(1) Hobbs, P. V. Ice Physics. (Oxford University Press, Oxford, 1974).

(2) Petrenko, V. \& Withworth R. Physics of Ice. (Oxford University Press, Oxford, 1999).

(3) Salzmann, C. G., Radaelli, P. G., Slaterc, B. \& Finney, J. L. The polymorphism of ice: five unresolved questions. Phys. Chem. Chem. Phys. 13, 18468 (2011).

(4) Salzmann, C. G. Advances in the experimental exploration of water's phase diagram. J. Chem. Phys. 150, 060901 (2019).

(5) Bartels-Rausch, T. et al. Ice structures, patterns, and processes: A view across the icefields. Rev. Mod. Phys. 84, 885 (2012).

(6) König, H. Eine kubische Eismodifikation. Z Kristallogr. 105, 279 (1943).

(7) Dowell L. G. \& Rinfret A. P. Low-temperature forms of ice as studied by x-ray diffraction. Nature 188, 1144 - 1148 (1960).

(8) Bertie, J. E., Calvert, L. D. \& Whalley, E. Transformations of Ice II, Ice III, and Ice V at Atmospheric Pressure. J. Chem. Phys. 38, 840 (1963).

(9) Bertie, J. E., Calvert, L. D. \& Whalley, E. Transformations of ice VI and ice VII at atmospheric pressure. Can. J. Chem. 42, 1373 (1964).

(10) Arnold G. P., Finch E. D., Rabideau S. W., \& Wenzel R. G. Neutron-diffraction study of ice polymorphs. III. Ice Ic. J. Chem. Phys. 49, 4354 - 4369 (1968).

(11) Klotz, S., Besson, J. M., Hamel, G., Nelmes, R. J. Loveday, J. S. \& Marshall, W. G. Metastable ice VII at low temperature and ambient pressure. Nature 398, 681 (1999).

(12) Murray, B. J., Knopf, D. A. \& Bertram A. K., The formation of cubic ice under conditions relevant to Earths atmosphere. Nature 434, 2230 (2005). 
(13) Falenty A., \& Kuhs, W. F. Self-preservation of $\mathrm{CO}_{2}$ gas hydrates - surface microstructure and ice perfection. J. Phys. Chem. B 113, 15975-15988 (2009).

(14) Falenty A., Hansen T., \& Kuhs, W. F. Cubic ice formation and annealing from $\mathrm{CO}_{2}$ clathrate hydrate decomposition at low temperatures. in Physics and Chemistry of Ice, eds Furukawa Y., Sazaki G., Uchida T., Watanabe N (Hokkaido Univ Press, Sapporo), 411- 419 (2011).

(15) Baker, J. M., Dore, J. C., \& Behrens, P. Nucleation of ice in confined geometry. J. Phys. Chem. B 101, 6226-6229 (1997).

(16) Kuhs, W. F., Sippel, C., Falenty, A. \& Hansen, T. C. Extent and relevance of stacking disorder in ice Ic. PNAS 109, 21259 (2012).

(17) Malkin, T. L., Murray, B. J., Brukhno, A. V., Anwar, J., \& Salzmann, C. G. Structure of ice crystallized from supercooled water. PNAS 109, 1041-1045 (2012).

(18) Malkin, T. L., Murray, B. J., Salzmann, C. G., Molinero, V., Pickering, S. J. \& Whale, T. F. Stacking disorder in ice I. Phys. Chem. Chem. Phys. 17, 60 (2015).

(19) Whalley, E. Scheiner's halo: evidence for ice Ic in the atmosphere. Science 211, 389-390 (1981).

(20) Murphy, D. M. Dehydration in cold clouds is enhanced by a transition from cubic to hexagonal ice. Geophys. Res. Lett. 30, 2230 (2003).

(21) Murray, B. J., Salzmann, C. G., Heymsfield, A J., Dobbie, S., Neely III, R. R., \& Cox, C. J. Trigonal ice Crystals in earth's atmosphere. Bull. Amer. Meteor. Soc. 94, 169-186 $(2015)$.

(22) Gronkowski, P. The search for a cometary outbursts mechanism: a comparison of various theories. Astronomische Nachrichten: Astronomical Notes 328, 126-136 (2007). 
(23) Hansen, T. C., Koza, M. M. \& Kuhs, W. F. Formation and annealing of cubic ice: I. Modelling of stacking faults. J. Phys.: Condens. Matter 20, 285104 (2008).

(24) del Rosso, L., Celli, M., \& Ulivi, L. A New Porous Water Ice Stable at Atmospheric Pressure Obtained by Emptying a Hydrogen Filled Ice. Nature Commun. 7, 13394 (2016).

(25) del Rosso, L., Grazzi, F., Celli, M., Colognesi, D., Garcia-Sakai, V., \& Ulivi, L. Refined structure of metastable ice XVII from neutron diffraction measurements. J. Phys. Chem. C 120, 26955-26959 (2016).

(26) del Rosso, L., Celli, M., Colognesi, D., Rudić S., English, N. J., Burnham, C. J., \& Ulivi, L. Dynamics of hydrogen guests in ice XVII nanopores. Phys. Rev. Mat. 1, 065602 (2017).

(27) Giacovazzo, C., Monaco, H. L., Viterbo, F., Scordari, G., Gilli, G., Zanotti, G., \& Catti, M. Fundamentals of crystallography. IUCr texts on crystallography, Oxford University Press (1992).

(28) Larson, A. C., \& Von Dreele, R. B. General Structure Analysis System (GSAS). Los Alamos Nat. Lab. Rep. LAUR 86, 748 (2004).

(29) Rodriguez-Carvajal, J. Recent advances in magnetic structure determination by neutron powder diffraction. Physica B 192, 55 (1993). The programs of the FullProf Suite can be obtained at http://www.ill.eu/sites/fullprof/.

(30) Kuhs, W. F., Bliss, D., \& Finney, J. High-resolution neutron powder diffraction study of ice Ic. Journal de Physique Colloques 48 (C1), 631-636 (1987).

(31) Hansen, T. C., Sippel, C. \& Kuhs, W. F. Approximations to the full description of stacking disorder in ice I for powder diffraction. Z. Kristallogr. 230, 75-86 (2015). 
(32) Playford, H. Y., Whale, T. F., Murray, B., Tucker, M. G. \& C. G. Salzmann, Analysis of stacking disorder in ice I using pair distribution functions. J. Appl. Cryst. 51, 1211-1220 (2018).

(33) Amaya, A. J., et al. How Cubic Can Ice Be?. J. Chem. Phys. Lett. 8, 3216 (2017).

(34) Röttger, K., Endriss, A., Ihringer, J., Doyle, S., \& Kuhs, W. F. Lattice Constants and Thermal Expansion of $\mathrm{H}_{2} \mathrm{O}$ and $\mathrm{D}_{2} \mathrm{O}$ Ice Ih Between 10 and 265 K. Acta Cryst. B 50, 644-648 (1994).

(35) Fortes, A. D. , Accurate and precise lattice parameters of $\mathrm{H}_{2} \mathrm{O}$ and $\mathrm{D}_{2} \mathrm{O}$ ice $\mathrm{Ih}$ between 1.6 and $270 \mathrm{~K}$ from high-resolution time-of-flight neutron powder diffraction data. Acta Cryst. B 74, 196-216 (2018).

(36) Treacy, M. M. J., Newsam, J. M., \& Deem, M. W. A general recursion method for calculating diffracted intensities from crystals containing planar faults. Proc. Roy. Soc. Lond. A 433499 - 520 (1991).

(37) Pimentel, G. C., \& Sederholm, C. H. Correlation of infrared stretching frequencies and hydrogen bond distances in crystals. J. Chem. Phys. 24, 639-641 (1956).

(38) Pruzan, P. Pressure effects on the hydrogen bond in ice up to 80 GPa. J. Mol. Struct. 322, 279-286 (1994).

(39) Vos, W. L., Finger, L. W., Hemley, R. J., \& Mao, H. K. Pressure dependence of hydrogen bonding in a novel $\mathrm{H}_{2}-\mathrm{H}_{2} \mathrm{O}$ clathrate. Chem. Phys. Lett. 257, 524-530 (1996).

(40) Carr, T. H. G., Shephard, J. J., \& Salzmann, C. G., Spectroscopic signature of stacking sisorder in Ice I. J. Phys. Chem. Lett. 5, 2469-2473 (2014).

(41) Komatsu, K., \& al. Ice Ic without stacking disorder by evacuating hydrogen from hydrogen hydrate. Preprint, arXiv:1909.03400 (2019). 
(42) Handa, Y. P., Klug, D. D., \& Whalley, E. Difference in energy between cubic and hexagonal ice. J. Chem. Phys. 84, 7009 (1986).

(43) Engel, E. A., Monserrat, B. \& Needs, R. J. Anharmonic nuclear motion and the relative stability of hexagonal and cubic ice. Phys. Rev. X 5, 021033 (2015).

(44) Raza, Z., Alfè, D., Salzmann, C. G., Klimeš, J., Michaelides, A. \& Slater, B. Proton ordering in cubic ice and hexagonal ice; a potential new ice phase - XIc. Phys. Chem. Chem. Phys. 13, 19788 (2011). 


\section{Table}

Table 1: Refined structural parameters of ice XVII and Ic as obtained by the fit of the neutron experimental data. Lattice constants $(a, c)$, atomic fractional coordinates $(x, y, z)$, displacement parameters $\left(B_{\text {iso }}\right)$ and site occupation (Occ.) obtained by means of Rietveld refinement of the diffraction patterns. Data were collected on HRPD (back scattering and equatorial banks, 30-130 ms time-of-flight window) before $(130 \mathrm{~K})$, during $(155 \mathrm{~K})$ and after $(170 \mathrm{~K})$ the transition between Ice XVII and Ice Ic, and on D20 at $160 \mathrm{~K}(\lambda=1.542 \AA)$.

\begin{tabular}{|c|c|c|c|c|c|c|c|c|c|c|c|c|}
\hline $\begin{array}{l}\text { Ice phase } \\
\text { (intrum.) }\end{array}$ & $\begin{array}{c}\mathrm{T} \\
{[\mathrm{K}]}\end{array}$ & $\begin{array}{l}R_{W} \\
{[\%]}\end{array}$ & $\begin{array}{l}\text { Space } \\
\text { group }\end{array}$ & $\begin{array}{c}a \\
{[\AA]}\end{array}$ & $\begin{array}{c}c \\
{[\AA]}\end{array}$ & Atom & Site & $x$ & $y$ & $z$ & $\begin{array}{l}B_{\text {iso }} \\
{\left[\AA^{2}\right]}\end{array}$ & Occ. \\
\hline $\begin{array}{c}\text { XVII } \\
\text { (HRPD) }\end{array}$ & 130 & 4.2 & $P 6_{1} 22$ & $6.33675(6)$ & $6.05714(9)$ & $\begin{array}{c}\mathrm{O} \\
\mathrm{D} 1 \\
\mathrm{D} 2\end{array}$ & $\begin{array}{c}6 b \\
12 c \\
12 c\end{array}$ & $\begin{array}{c}0.23402(13) \\
0.3408(3) \\
0.0642(3)\end{array}$ & $\begin{array}{c}2 x \\
0.5720(3) \\
0.3706(3)\end{array}$ & $\begin{array}{c}1 / 4 \\
0.3731(4) \\
0.3080(3)\end{array}$ & $\begin{array}{l}1.81(3) \\
2.41(4) \\
2.63(4)\end{array}$ & $\begin{array}{l}1.0 \\
0.5 \\
0.5\end{array}$ \\
\hline $\begin{array}{l}\text { XVII } \\
\text { and } \\
\text { Ic } \\
\text { (HRPD) }\end{array}$ & 155 & 4.3 & $P 6_{1} 22$ & $6.34463(12)$ & $6.0611(2)$ & $\begin{array}{c}\mathrm{O} \\
\mathrm{D} 1 \\
\mathrm{D} 2 \\
\mathrm{O} \\
\mathrm{D}\end{array}$ & $\begin{array}{c}6 b \\
12 c \\
12 c \\
8 b \\
32 e\end{array}$ & $\begin{array}{c}0.2342(3) \\
0.3410(6) \\
0.0649(7) \\
3 / 8 \\
0.4665(2)\end{array}$ & $\begin{array}{c}2 x \\
0.5712(5) \\
0.3707(5) \\
3 / 8 \\
x\end{array}$ & $\begin{array}{c}1 / 4 \\
0.3726(7) \\
0.3080(6) \\
3 / 8 \\
x\end{array}$ & $\begin{array}{c}2.02(6) \\
2.41(8) \\
2.80(11) \\
2.92(8) \\
2.73(9)\end{array}$ & $\begin{array}{l}1.0 \\
0.5 \\
0.5 \\
\\
1.0 \\
0.5\end{array}$ \\
\hline $\begin{array}{c}\text { Ic } \\
(\mathrm{D} 20)\end{array}$ & 160 & 4.1 & $F d \overline{3} m$ & $6.37912(13)$ & - & $\begin{array}{l}\mathrm{O} \\
\mathrm{D}\end{array}$ & $\begin{array}{c}8 b \\
32 e\end{array}$ & $\begin{array}{c}3 / 8 \\
0.46591(11)\end{array}$ & $\begin{array}{c}3 / 8 \\
x\end{array}$ & $\begin{array}{c}3 / 8 \\
x\end{array}$ & $\begin{array}{l}2.59(3) \\
3.06(4)\end{array}$ & $\begin{array}{l}1.0 \\
0.5\end{array}$ \\
\hline $\begin{array}{c}\text { Ic } \\
(\mathrm{HRPD})\end{array}$ & 170 & 4.4 & $F d \overline{3} m$ & $6.37451(9)$ & - & $\begin{array}{l}\mathrm{O} \\
\mathrm{D}\end{array}$ & $\begin{array}{c}8 b \\
32 e\end{array}$ & $\begin{array}{c}3 / 8 \\
0.46608(9)\end{array}$ & $\begin{array}{c}3 / 8 \\
x\end{array}$ & $\begin{array}{c}3 / 8 \\
x\end{array}$ & $\begin{array}{l}2.20(3) \\
2.54(3)\end{array}$ & $\begin{array}{l}1.0 \\
0.5\end{array}$ \\
\hline
\end{tabular}




\section{Figure captions}

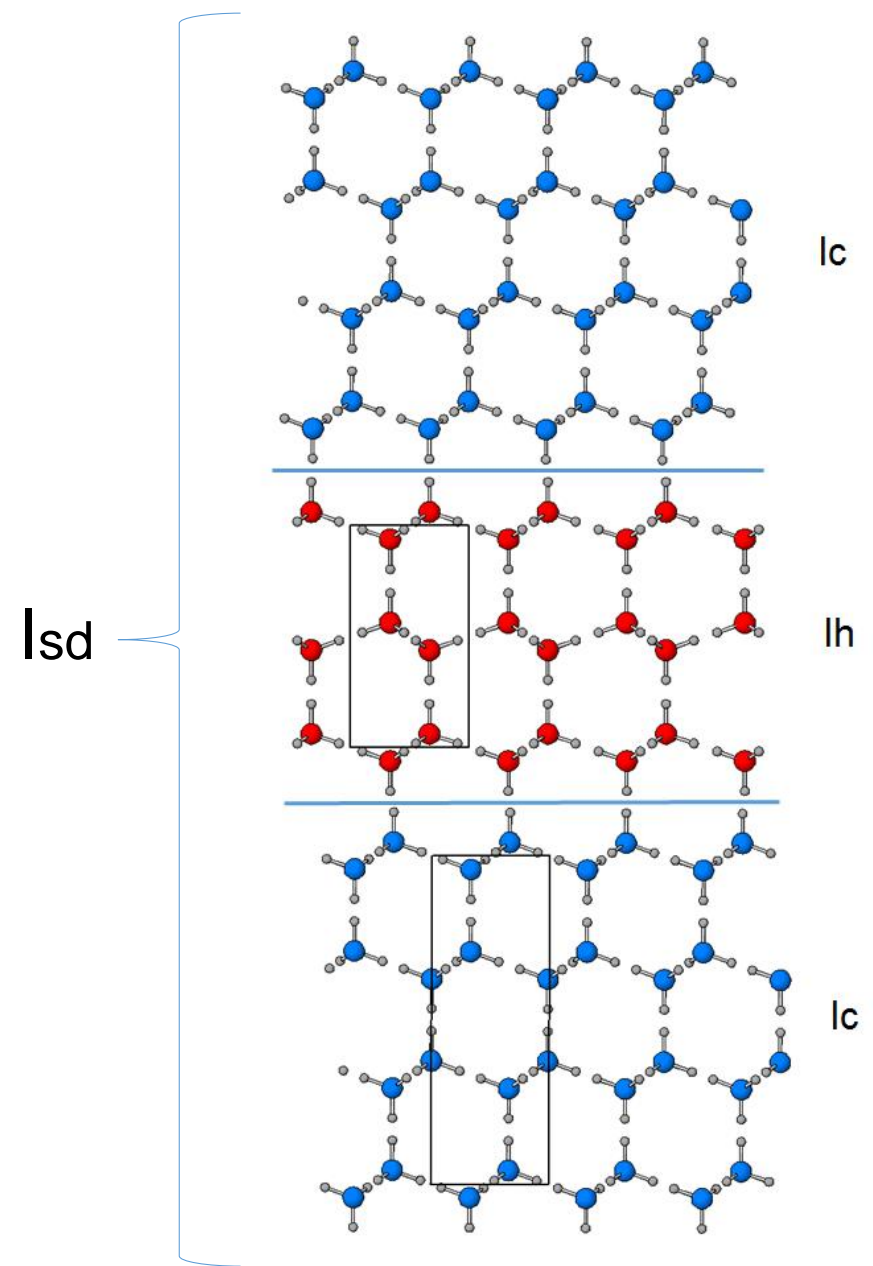

Figure 1: Pictorial representation of the atomic positions of ice Isd. In this example a hexagonal slice of Ih phase is inserted into a cubic Ic matrix, sketching typical defects of ice Isd. Atoms are drawn as blue (Ic) or red (Ih) balls for $\mathrm{O}$ and grey balls for disordered H. In the hexagonal and cubic cases the projection planes are $\left(\begin{array}{lll}2 & -1 & 0\end{array}\right)$ with vertical axis [001], and (-1 10$)$ with vertical [111], respectively. Unit cells are outlined with hexagonal setting also for cubic ice, for the sake of comparison. 

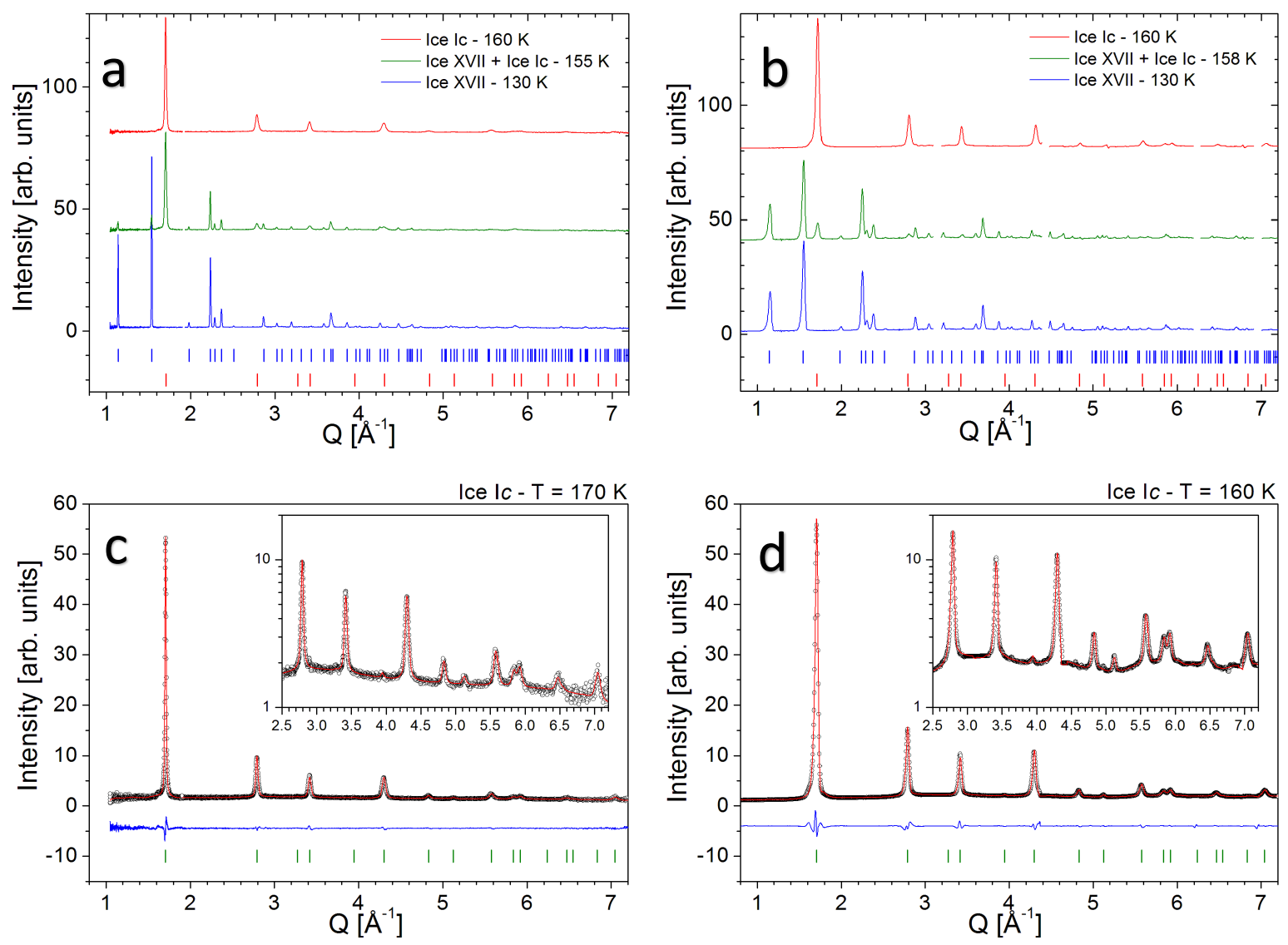

Figure 2: Diffraction patterns measured at ISIS (left) and at the ILL (right). In the top panels, some representative diffraction patterns are plotted against $Q=2 \pi / d$, clearly showing the transition from ice XVII (blue) to ice Ic (red). Lower panels report the Rietveld fits (red line) of two diffraction patterns (black circles) recorded for ice Ic at $170 \mathrm{~K}$ on HRPD (c) and at $160 \mathrm{~K}$ on D20 (d), both plotted as a function of $Q$. The residuals (blue line) and the positions of the ice Ic reflections (green bars) are shown in each panel. To improve the visualization and show the high fit quality in all the $Q$ range, a semilog plot with an enlargement of the high $Q$ zone is reported in the insets. 


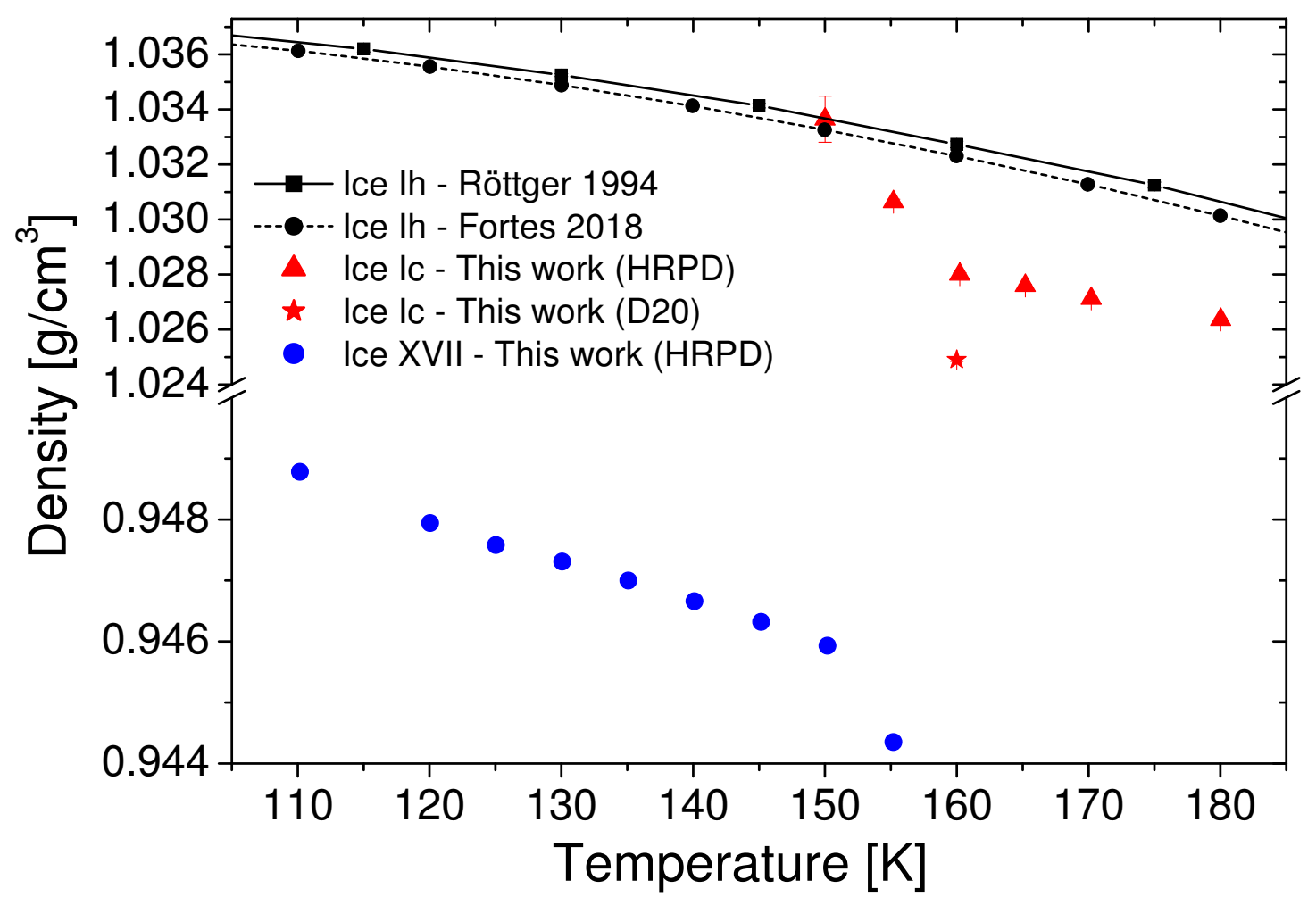

Figure 3: Density of ice XVII and ice Ic as a function of temperature. Density of $\mathrm{D}_{2} \mathrm{O}$ samples is reported in $\mathrm{g} / \mathrm{cm}^{3}$ for ice XVII (blue dots) and ice Ic (red triangles, HRPD data, red star, D20 datum). The values are obtained by means of Rietveld refinement of the diffraction data. For comparison, the density of $\mathrm{D}_{2} \mathrm{O}$ ice Ih as obtained from a synchrotron X-ray ${ }^{34}$ and neutron ${ }^{35}$ measurements are reported in the plot with black symbols and lines. 


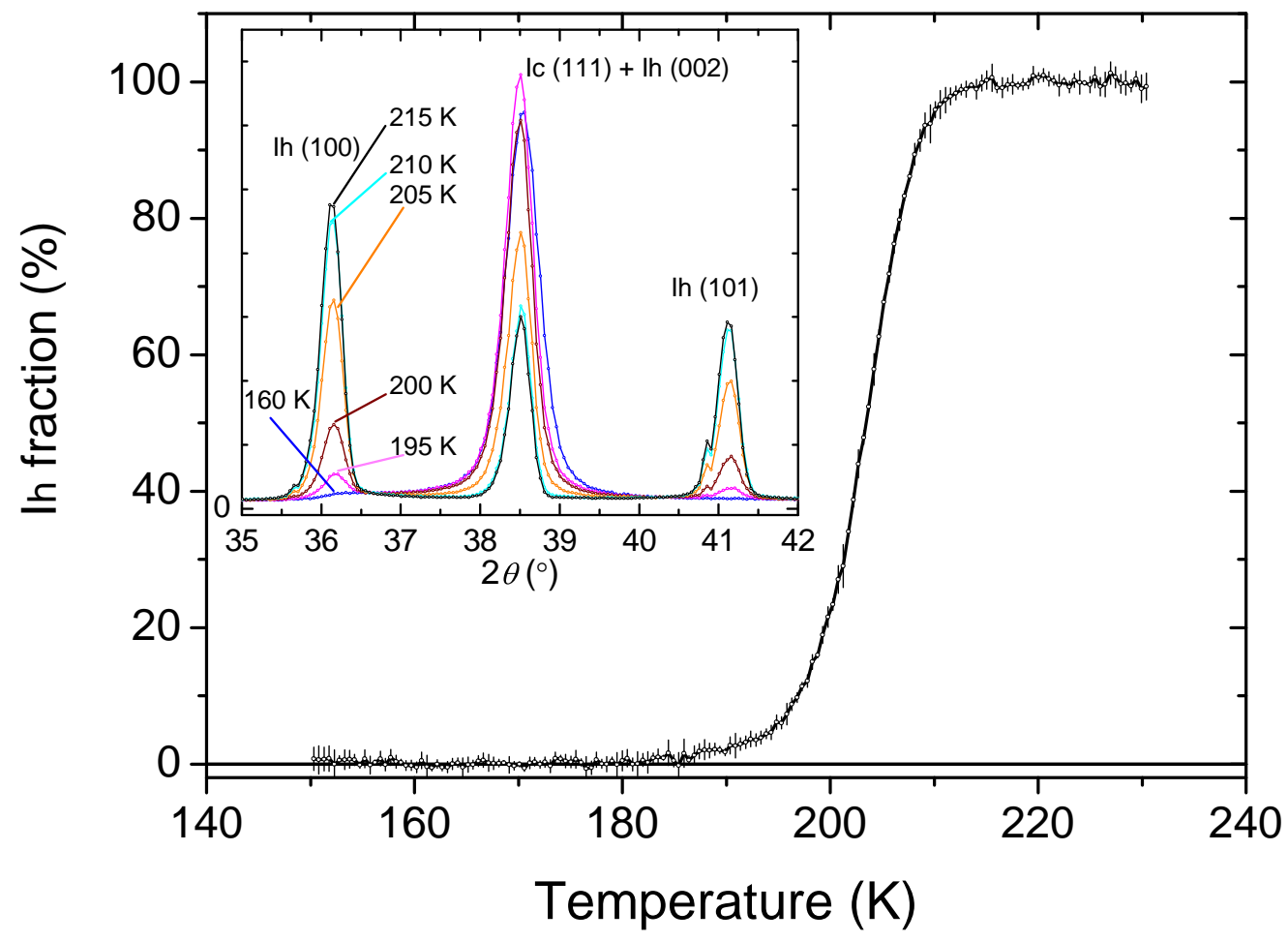

Figure 4: Transformation of ice Ic into ice Ih. Ice Ih fraction (\%) as a function of temperature, obtained from the data collected on D20 at $\lambda=2.42 \AA$. This is calculated (see also Method section) as the average of the intensity ratio of five Ih Bragg reflections (namely (100), (101), (102), (103), (112)), assuming that the sample is cubic at low temperature $(150 \mathrm{~K})$ and hexagonal at high temperature $(220 \mathrm{~K})$. A few diffraction patterns in the $2 \theta$ range of the (111) reflection of cubic ice are shown in the inset. 

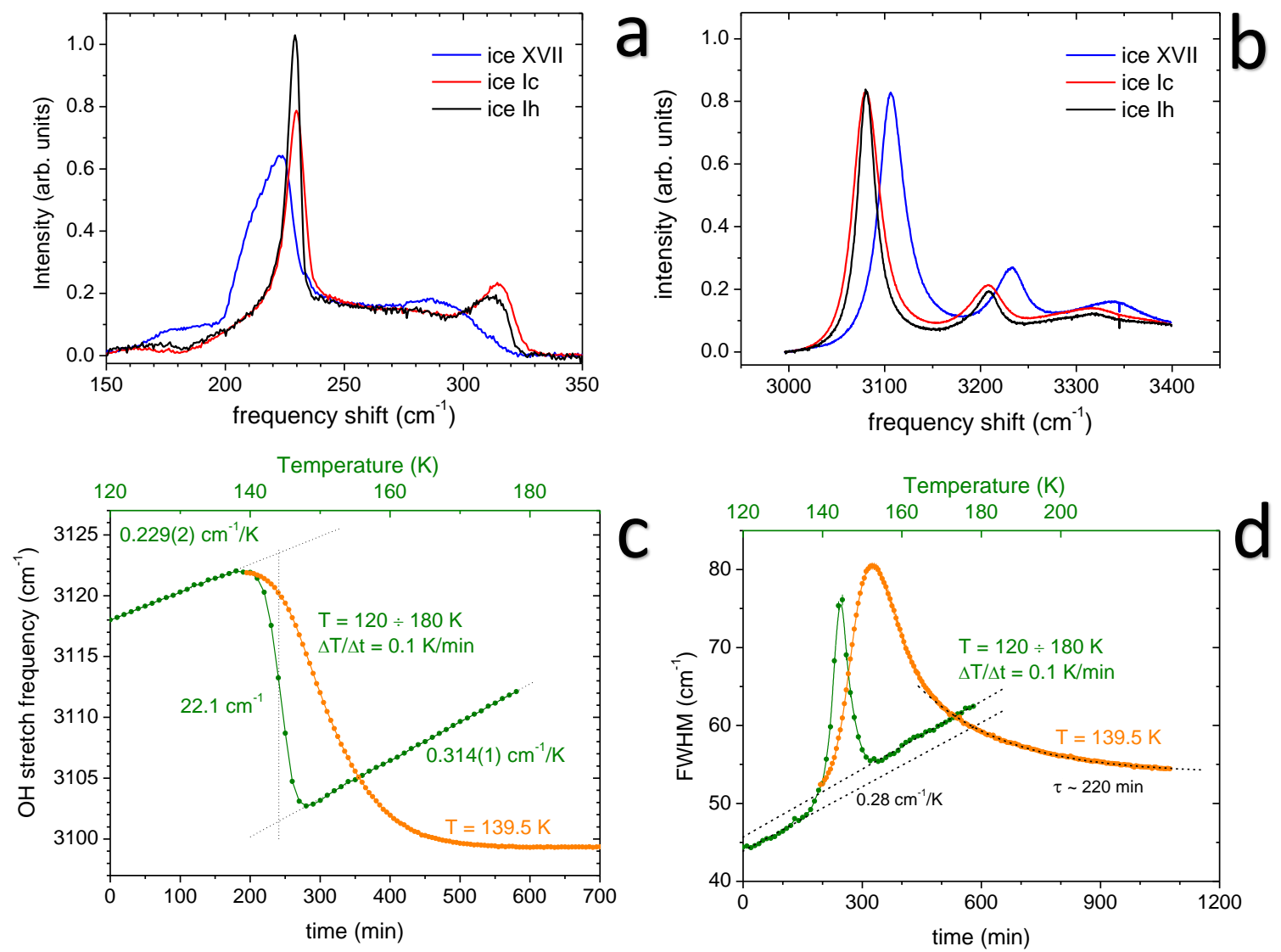

Figure 5: Raman characterization of the transition from ice XVII to ice Ic. Raman spectra of the three phases of ice considered in this study, ice XVII (blue), ice Ic (red) and ice Ih (black), in the lattice modes (a) and $\mathrm{OH}$-stretching frequency region (b), measured at $50 \mathrm{~K}$. Differences are evident between ice XVII and the other two, and are very small between ice Ic and Ih. Frequency position (c) and width (d) of the $\mathrm{OH}$-stretching band (centre and width of the Lorentzian curve fitting the major band) during the transition ice XVII - ice Ic, plotted as a function of both time (lower black axis) and temperature (upper green axis), while performing a $0.1 \mathrm{~K} / \mathrm{min}$ temperature ramp (green line with dots). The same quantity recorded at constant temperature $(T=139.5 \mathrm{~K})$ is plotted as a function of time only (orange line with dots). 


\section{Methods}

\section{Sample preparation}

Our starting material is the hydrogen filled ice $\left(\mathrm{D}_{2} \mathrm{O}\right.$ for the neutron experiment or $\mathrm{H}_{2} \mathrm{O}$ for the Raman one) in the $\mathrm{C}_{0}$-phase. The high pressure synthesis procedure, with the recovery of the sample at ambient pressure, either for Raman spectroscopy or neutron diffraction measurements is similar to that described in Ref. 25. The sample is transferred from the Dewar into the experimental (Raman or neutron) cell at liquid nitrogen temperature in a dry nitrogen atmosphere to prevent the condensation on the sample surface of the water present in the air. Once into the cryostat, the pristine $\mathrm{H}_{2}$ filled ice sample underwent the so called annealing treatment, i.e. heated up to $110 \mathrm{~K}$ under dynamic vacuum for about 1 hour, to remove all the guest molecules trapped inside the water structure, and thus obtaining the pure ice XVII.

\section{Raman spectroscopy}

For the Raman experiment we have used the apparatus described in Ref. 45, equipped with a Raman cell with a single round window on the top surface. The sample handling and transfer into the cell and the annealing procedure are described above. The temperature of the sample cell is controlled with a reproducibility of $0.1 \mathrm{~K}$. Few tens of millibar of Ar gas helps to reach thermal equilibrium between the powder sample and the cell. The laser beam (Ar ion laser at $\lambda=5145 \AA$ ) having a power on the sample of about $10 \mathrm{~mW}$, is focussed onto a $80 \mu \mathrm{m}$ spot on the powder sample, laying on a horizontal surface in the cell, in an almost back-scattering geometry. The scattered light is collected in a vertical direction, and is focussed onto the entrance slit of a monochromator, equipped with a cooled CCD detector. Spectra are collected in 30 or $10 \mathrm{~min}$, for the lattice modes or the OH-stretching region, respectively. The data reported in Fig. $5 \mathbf{c}$ and $5 \mathbf{d}$ are obtained by using a ramp on the temperature controller and by means of an automatic spectra collection and analysis. The 
frequency axis is calibrated by means of a Ne spectral lamp with an accuracy of $0.5 \mathrm{~cm}^{-1}$, which is also the accuracy of the position and width of the OH-stretching peak discussed in the text. Examples of experimental spectra recorded during the ice XVII - ice Ic transition are reported in the SI (Supplementary Note 5).

\section{Neutron diffraction}

The time-of-flight measurements performed with the HRPD at ISIS (Rutherford Appleton laboratory, U.K.) were based on three detector banks at high, middle and low resolution $\left(2 \theta=168.3^{\circ}, 90^{\circ}, 30^{\circ}, \Delta d / d \sim 6 \cdot 10^{-4}, 2 \cdot 10^{-3}, 2 \cdot 10^{-2}\right.$ respectively). Data were collected in the $30-130$ and $100-200$ ms time-of-flight windows covering the $0.65-10.2 \AA$ and $2.15-15.7 \AA d$ spacing ranges, respectively, with all three banks. Data are available at the ISIS database. ${ }^{46}$ As sample container, we used a cylindrical vanadium cell provided with a capillary connected to a gas manifold equipped with a vacuum pump and various gas cylinders, that allows to set the pressure inside the cell in the range between 0 and 10 bar. No empty cell measurement was needed since the vanadium container gives a negligible coherent contribution to the scattering. Low temperatures were achieved with a closed-cycle refrigerator mounted in HRPD's sample tank.

Raw data were time-focused, normalised to the incident spectrum and corrected for instrumental efficiency using a V:Nb standard with the suite of diffraction routines in Mantid. ${ }^{47}$ Data were analysed by the Rietveld refinement ${ }^{27}$ with the GSAS-I software package ${ }^{28}$, using data collected on the two detector banks at $2 \theta=168.3^{\circ}$ (30-130 ms t.o.f. window) and $90^{\circ}$ (30-130 and 100-200 ms t.o.f. windows). The HRPD instrument calibration file, used to convert and fit the raw data from time-of-flight to $d$-spacing, was carefully obtained by measuring a NIST silicon standard (SRM640e) in the same cycle of our experiment. A peak profile function 3, i.e. a convolution of back-to-back exponentials with a pseudo-Voigt function, was used for fitting the data: the profile parameters $\alpha-0, \beta-0$ and $\beta-1$, that model the instrumental broadening of the diffraction peaks, were carefully determined by measuring a 
$\mathrm{CeO}_{2}$ sample in the same cycle and were kept fixed during the refinement of the ice XVII experimental data; while the other Gaussian and Lorentzian profile parameters, that are mainly related to the peak broadening induced by the sample, were always refined. In the HRPD experiment, the time interval needed to perform one measurement for a given thermodynamic point was about one hour, including about 30 minutes for an effective sample thermal equilibration, and 15 minutes for each of the two time-of-flight windows acquired.

Two similar experiment were also performed by means of the high-flux high-resolution D20 diffractometer present at the ILL. In the first experiment the aluminum sample cell was connected to a vacuum pump, and the thermodynamic treatment of the sample has been quite similar to the HRPD experiment. In this case, short measurements were performed every $10 \mathrm{~K}$ in the range $110-160 \mathrm{~K}$, leaving 15 minutes for the stabilization at every temperature change. Other technical details related to this experiment and data analysis are reported in Ref. 48, and data are stored in the appropriate ILL database. ${ }^{49}$ The second experiment on the D20 diffractometer was performed to study the transition from ice Ic to ice Ih. ${ }^{50}$ Ice Ic was prepared from ice XVII in the IFAC laboratories (Italy), loaded in a sealed cylindrical vanadium can and shipped to ILL. The data were analysed by means of Rietveld refinement, but, as discussed in the text, the ice Ih fraction as a function of temperature, $F_{h}(T)$, has been estimated as $F_{h}(T)=\left\langle\left(I_{h k l}(T)-I_{h k l}\left(T_{1}\right)\right) /\left(I_{h k l}\left(T_{2}\right)-I_{h k l}\left(T_{1}\right)\right)\right\rangle$, where $I_{h k l}(T)$ is the intensity of the $(h k l)$ reflection at the temperature $T$, the angular brackets indicate the average over the five reflections (100), (101), (102), (103), (112), $T_{1}=150 \mathrm{~K}$ and $T_{2}=220 \mathrm{~K}$.

\section{Data Availability}

The experimental data presented in this work are available form the DOI reported in Ref. 46, 49 and 50 and from the corresponding authors upon reasonable request. 


\section{References}

(45) Giannasi, A., Celli, M., Grazzi, F., Ulivi, L., \& Zoppi, M. An apparatus for simultaneous thermodynamic and optical measurements with large temperature excursions. Rev. Sci. Instrum. 79, 13105 (2008).

(46) Ulivi, L., Grazzi, F., Colognesi, D., del Rosso, L., \& Celli, M. Experiment RB1820334. data are available at DOI 10.5286/ISIS.E.RB1820334.

(47) Arnold, O., et al., Mantid - Data analysis and visualization package for neutron scattering and $\mu$ SR experiments. Nucl. Instrum. Meth. A 764, 156 (2014).

(48) Catti, M., del Rosso, L., Ulivi, L., Celli, M., Grazzi, F. \& Hansen, T. C. Ne- and $\mathrm{O}_{2^{-}}$ filled ice XVII: a neutron diffraction study. Phys. Chem. Chem. Phys. 21, 14671-14677 (2019).

(49) Ulivi, L., Catti, M., Celli, M., Colognesi, D., del Rosso, L., Grazzi, F. \& Hansen, T. C. 2018, DOI 10.5291/ILL-DATA.5-22-759.

(50) Ulivi, L. and Hansen, T. C. 2018, DOI 10.5291/ILL-DATA.EASY-498 